\title{
Parathyroid Gland Lipoadenoma
}

National Cancer Institute

\section{Source}

National Cancer Institute. Parathyroid Gland Lipoadenoma. NCI Thesaurus. Code C48283.

A parathyroid gland adenoma that contains mature adipocytes. 